\title{
On Finding Another Room-Partitioning of the Vertices
}

\author{
Jack Edmonds ${ }^{1}$ \\ Equipe Combinatoire 83 Optimisation \\ Université Pierre et Marie Curie \\ Paris, France \\ Laura Sanità ${ }^{2}$ \\ Institute of Mathematics \\ Ècole Polytechnique Fèdèrale de Lausanne \\ Lausanne, Switzerland
}

\begin{abstract}
Let $T$ be a triangulated surface given by the list of vertex-triples of its triangles, called rooms. A room-partitioning of $T$ is a subset $R$ of the rooms such that each vertex of $T$ is in exactly one room in $R$.

We prove that if $T$ has a room-partitioning $R$, then there is another roompartitioning of $T$ which is different from $R$. The proof is a simple algorithm which walks from room to room, which however we show to be exponential by constructing a sequence of (planar) instances, where the algorithm walks from room to room an exponential number of times relative to the number of rooms in the instance.
\end{abstract}

We unify the above theorem with Nash's theorem stating that a 2-person game has an equilibrium, by proving a combinatorially simple common generalization.

Keywords: Room-partitioning, Exchange algorithm, 2-person games. 


\section{Introduction}

Many Existentially Polytime (EP) theorems [1] have existence proofs that do not immediately imply a polynomial-time algorithm for finding what is asserted to exist. Although a lot of EP theorems also have polynomial-time algorithmic proofs, some of them have resisted effort to find polynomial-time algorithms. In particular, some of such EP theorems can roughly take the following form: "For any $T$, and $R$ in $T$, there is another different $R$ in $T$ ", as a consequence of some algorithmic techniques proving that "for any $T$, there is an even number of such $R$ in $T$ ". A powerful technique to prove these statements is showing that they are implied by vertex-degree parity in large implicit "exchange graphs" [2].

One of the most famous problem for which polynomial-time algorithms seem elusive, is finding a Nash equilibrium for 2-person games, despite its existence proof. In fact, the problem has been shown by Chen and Deng [3] to be complete for one important class of search problems, namely the class PPAD, identified in a seminal paper of Papadimitriou [7].

Elaborating on the above concepts, in this paper we prove that for for any triangulated surface, and any subset of its triangles partitioning its vertices, there exists another different subset of triangles partitioning the vertices. The proof is a simple "exchange algorithm" which however we show to have a running time (on a sequence of planar instances) that is exponential in the number of triangles. We then define an abstract generalization of such theorem which in fact generalizes the existence of an equilibrium for 2-person games and the Lemke-Howson algorithm [5] for finding such equilibria.

\section{The room-partitioning problem}

A rank-r simplicial pseudo manifold $(V, M)$ is a finite set $V$ of elements called the vertices and a family $M$ of subsets of $V$, called the rooms, each one of size $r$ and with the following property: every vertex subset of $V$ of size $r-1$ is contained in exactly 0 rooms or in exactly 2 rooms. The latter subsets are called the walls of $(V, M)$. A room-partitioning of $(V, M)$ is a subset $R$ of the rooms such that each vertex of $V$ is in exactly one room in $R$.

Theorem 2.1 Suppose that a manifold $(V, M)$ has a room-partitioning $R$. Then there is another room-partitioning $R^{\prime}$ of $(V, M)$, different from $R$.

1 Email: jackedmonds@rogers.com

2 Email: laura.sanita@epfl.ch 
Proof. Choose any vertex, say $w$, to be special. A $w$-skew room-family of $(V, M)$ is a subset $S$ of the rooms $M$ such that: (i) no room in $S$ contains the vertex $w$, (ii) some vertex $v$ is in exactly two rooms of $S$, (iii) every other vertex besides $v$ and $w$ is in exactly one room of $S$. Consider the so-called exchange graph $X$ determined by $(V, M)$ and $w$, as follows. The nodes of $X$ are all the room-partitionings of $(V, M)$ plus all the $w$-skew room-families of $(V, M)$. Two nodes of $X$ are joined by an edge of $X$ when each is obtained from the other by replacing one room by another. It is easy to see that the connected components of $X$ are simple paths and cycles. Still, the path ends are the room-partitionings of $(V, M)$. The result then follows observing that $X$ must have an even number of odd-degree nodes.

Given a room-partitioning $R$ of $(V, M)$, the exchange algorithm finds another room-partitioning $R^{\prime}$ of $(V, M)$, by simply walking along the path in $X$ from the node of $X$ corresponding to $R$ to the other end of its path. Going from one node to another by an edge of $X$ is called a pivot. Of course, the algorithm applied to $R^{\prime}$ takes us in reverse direction on the same path to $R$.

We now prove that the exchange algorithm is not a polynomial-time algorithm, by constructing a sequence of instances where the algorithm walks from room to room an exponential number of times, relative to the number of rooms. Each instance is a rank-3 manifold described by a triangulated surface, where a room corresponds to a vertex-triple of its triangles.
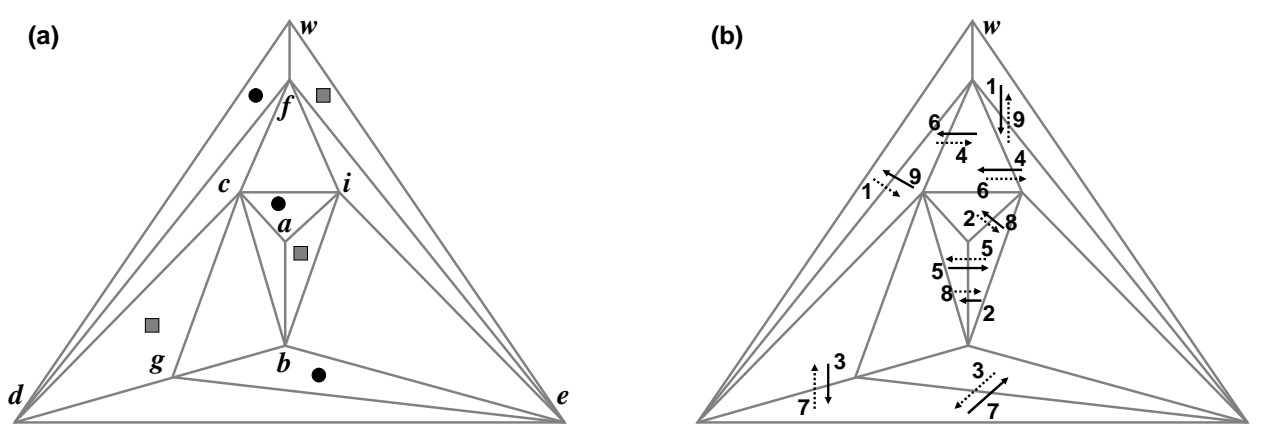

Fig. 1. The manifold $\left(V_{0}, M_{0}\right)$ in (a). In (b), bold and dashed arrows indicate the pivots made by the exchange algorithm to get from $R_{0}$ to $R_{0}^{\prime}$ and from $R_{0}^{\prime}$ to $R_{0}$, with the chosen special vertex $w$. As an example, bold arrow labelled by 1 has tail in room wef and head in room fie: it indicates that, in the first pivot (to get from $R_{0}$ to $R_{0}^{\prime}$ ) room $w f e$ is replaced by room fie.

Let $\left(V_{0}, M_{0}\right)$ be the manifold depicted in Fig. 1(a), where the gray squares 
indicate the triangles (rooms) corresponding to the initial room-partitioning $R_{0}$, and the black circles indicate the second room-partitioning $R_{0}^{\prime}$, which you get by applying the exchange algorithm to $R_{0}$, with the chosen special vertex $w$. In Fig. 1(b), bold arrows indicate in order the 9 pivots of the algorithm to get from $R_{0}$ to $R_{0}^{\prime}$. Dashed arrows indicate in order the 9 pivots of the algorithm to get from $R_{0}^{\prime}$ to $R_{0}$. Note that, each of these two algorithm applications is, of course, the reverse of the other.

To get example $\left(V_{1}, M_{1}\right)$ and $R_{1}$ from $\left(V_{0}, M_{0}\right)$ and $R_{0}$, consider the triangulation in Fig. 1 and do the following steps: (i) add a vertex $h$ along the edge $b c$ in the picture, (ii) add the edges $h g$ and $h a$, (iii) replace the triangle $h a b$ with a shrunken copy of the big triangulated triangle $d f e$ (see Fig. 2).

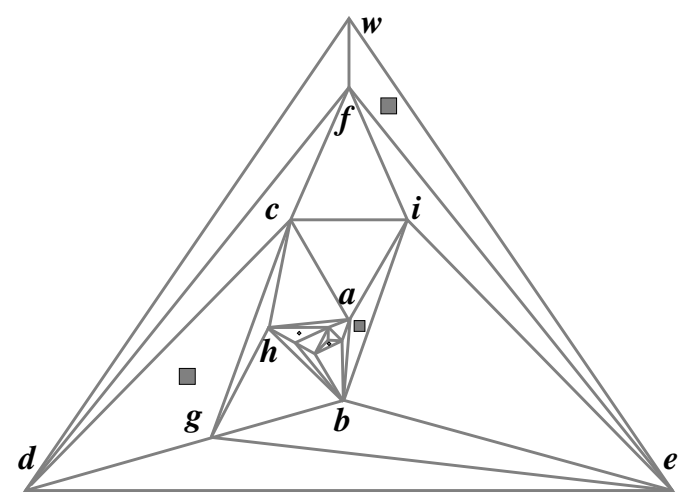

Fig. 2. The manifold $\left(V_{1}, M_{1}\right)$. Gray squares indicate the triangles corresponding to the initial room-partitioning $R_{1}$.

We get triangulated surface $\left(V_{k}, M_{k}\right)$ with room-partitioning $R_{k}$ from triangulated surface $\left(V_{k-1}, M_{k-1}\right)$ with room-partitioning $R_{k-1}$, applying the same steps as in the preceding paragraph. Next theorem easily follows from the picture of the pivots.

Theorem 2.2 For every positive integer $k,\left(V_{k}, M_{k}\right)$ has 12 more rooms than $\left(V_{k-1}, M_{k-1}\right)$. The exchange algorithm does $8 \cdot 2^{k}$ more pivots for $R_{k}$ in $\left(V_{k}, M_{k}\right)$ than for $R_{k-1}$ in $\left(V_{k-1}, M_{k-1}\right)$.

Corollary 2.3 The exchange algorithm for the room-partitioning problem has a running time that is exponential in the number of rooms.

We do not know an algorithm for finding a room-partitioning $R^{\prime}$, given a triangulated surface $T$ and an initial room-partitioning $R$, which is polynomialtime relative to the number of triangles in $T$. 
In the next sections, we unify the room-partitioning theorem 2.1 with Nash's theorem stating that a 2-person game has an equilibrium, by giving a combinatorially simple common generalization.

\section{Nash equilibria for 2-person games}

A bimatrix game (i.e., a 2-person game) is given by two bounded non-degenerate systems, $[A I] y=\mathbf{1}$ and $[I B] x=\mathbf{1}$. (The identity submatrices $I$ in the above systems are not the same but are appropriate to non-negative matrix $A$ and to non-negative matrix $B$, respectively.)

Let $R$ (resp. $T$ ) be the index set for the rows of $A$ and for the columns of $B$ (resp. for the rows of $B$ and for the columns of $A$ ). $R$ (resp. T) is called the set of pure strategies for Player 1 (resp. Player 2). When Player 1 plays a probability distribution, $y^{R}$ on $R$, and Player 2 plays a probability distribution, $x^{T}$ on $T$, the expected pay-off for Player 1 is $A$ pre-multiplied by $y^{R}$ and post-multiplied by $x^{T}$; the expected pay-off for Player 2 is $B$ premultiplied by $x^{T}$ and post-multiplied by $y^{R}$. The pair $\left(x^{T}, y^{R}\right)$ is called an equilibrium if neither player can improve his expected payoff by unilaterally changing his probability distribution.

Let $V$ be the column-index set of matrix $[A I]$ and of matrix $[I B]$, that is, $V$ is the disjoint union of $R$ and $T$. $R$ is a feasible basis of system $[A I] y=\mathbf{1}$ and $T$ is a feasible basis of system $[I B] x=1$. (A feasible basis is a maximal independent subset of columns such that the solution obtained by setting equal to zero the variables corresponding to columns not in the basis, is nonnegative.) In particular, $(R, T)$ is a complimentary pair of feasible bases, since $R=V-T$ and vice versa. It is a beautiful easy-to-prove key theorem that:

Theorem 3.1 The equilibria of the game are given by the complimentary pairs of feasible bases of $[A I] y=\mathbf{1}$ and $[I B] x=\mathbf{1}$, other than $(R, T)$.

Hence the following Theorem 3.2 is the crucial part of an algorithmic proof of Nash's theorem that every bimatrix game has an equilibrium.

Theorem 3.2 There is another complimentary pair of feasible bases of $[A I] y=$ 1 and $[I B] x=\mathbf{1}$, other than the given starting pair $(R, T)$.

We now prove an abstract version of Theorem 3.2, relying on the definition of manifolds. Before, we need a theorem implicit in the simplex method for linear programming:

Theorem 3.3 (The Simplex Theorem) Let $A y=b$ be a non-degenerate 
and bounded system. For any feasible basis $T$ and any column $j$ not in $T$, there is a unique column $i$ in $T$, such that $T^{\prime}=(T+j-i)$ is a feasible basis.

Another way of saying Theorem 3.3 is:

Theorem 3.4 If $V$ is the column-set of $A$, and the members of $M$ are the sets $R=V-T$ such that $T$ is a feasible basis of $A$, then, where $n$ is the number of columns of $A$ and $m$ is the rank of $A,(V, M)$ is a rank- $(n-m)$ manifold.

A simplicial manifold $(V, M)$ is the combinatorial type of a geometric simplicial polytope (the convex hull of a finite set of points such that every face is a geometric simplex) if and only if $(V, M)$ can be realized as in Theorem 3.3. In fact, a manifold with many rooms might be described implicitly by a non-degenerate system of linear equations having a bounded non-negative solution-set.

We are now ready to state the Abstract Equilibrium Theorem:

Theorem 3.5 (Abstract Equilibrium Theorem) Let $\left(V, M_{1}\right)$ and $\left(V, M_{2}\right)$ be manifolds both with vertex-set $V$ and, respectively, with room-sets $M_{1}$ and $M_{2}$. Then there is an even number of complimentary partitions of $V$ into a room $T_{1}$ of $M_{1}$ and a room $T_{2}$ of $M_{2}$ (that is, such that $T_{2}=V-T_{1}$ ).

Proof. Choose an element $w$ in $V$. Let $X$ be the graph whose nodes are pairs $\left(T_{1}, T_{2}\right)$ such that $T_{1}$ is a room of $M_{1}$ and $T_{2}$ is a room of $M_{2}$, and such that either $\left(T_{1}, T_{2}\right)$ is complimentary, or else non-complimentary such that vertex $w$ is in neither $T_{1}$ or $T_{2}$ and some one vertex $j$ is in both $T_{1}$ and $T_{2}$. Two nodes $\left(T_{1}, T_{2}\right)$ and $\left(T_{1}^{\prime}, T_{2}^{\prime}\right)$ of graph $X$ are joined by an edge in $X$ when $T_{1}=T_{1}^{\prime}$ and $T_{2}^{\prime}=T_{2}-j+i$ for some vertex $i$ in $\left(T_{1}-j\right) \cup\{w\}$, or when $T_{2}=T_{2}^{\prime}$ and $T_{1}^{\prime}=T_{1}-j+i$ for some vertex $i$ in $\left(T_{2}-j\right) \cup\{w\}$. Each complimentary pair $\left(T_{1}, T_{2}\right)$ is a node of $X$ which is in exactly one edge of $X$. Each non-complimentary node $\left(T_{1}, T_{2}\right)$ of $X$ is in exactly two edges of $X$.

Note that, using the Simplex Theorem 3.3, Theorem 3.5 clearly has Theorem 3.2 as an instance.

The Lemke-Howson algorithm [5] to find an equilibrium for a 2-person game is to make simplex pivots which walk along the path in graph $X$ from the node of $X$ corresponding to the starting complimentary pair of bases to the other end of the path. Savani and von Stengel [8] (see also [6]) have shown that the Lemke-Howson algorithm is exponential relative to the size of the matrices defining the game, not necessarily exponential relative to the number of simplices defined by the matrices. 


\section{Unifying Theorem}

We now give a common generalization of the Room-Partitioning Theorem 2.1 and the Abstract Equilibrium Theorem 3.5.

Let $M=\left[\left(V, M_{i}\right): i=1, \ldots, h\right]$ be an indexed collection of manifolds (which we call a manifold-family) all on the same vertex-set $V$. The manifolds of $M$ are not necessarily of the same dimension. Of course, all of them may be the same manifold. A room-family, $R=\left[R_{i}: i=1, \ldots, h\right]$, for manifold-family $M$, is where, for each $i, R_{i}$ is a room of manifold $\left(V, M_{i}\right)$ (i.e., a member of $M_{i}$ ). A room-partitioning $R$ for $M$ means a room-family whose rooms partition $V$.

Theorem 4.1 Given a manifold-family $M$ and a room-partitioning $R$ for $M$, there exists another different room-partitioning for $M$. In fact, for any manifold-family $M$, there is an even number of room-partitionings.

Proof. Choose a vertex, say $w$, to be special. A w-skew room-family for manifold-family $M$ means a room-family, $R=\left[R_{i}: i=1, \ldots, h\right]$ for $M$ such that $w$ is not in any of the rooms $R_{i}$, some vertex $v$ is in exactly two of the $R_{i}$, and every other vertex is in exactly one of the $R_{i}$. Consider the exchange-graph $X$, determined by $M$ and $w$, where the nodes of $X$ are all the room-partitionings for $M$ and all the $w$-skew room-families for $M$. Two nodes of $X$ are joined by an edge of $X$ when each is obtained from the other by replacing one room by another. It is easy to see that the room-partitionings for $M$ are all one-degree nodes of $X$, and the $w$-skew room-families for $M$ are two-degree nodes in $X$. Hence $X$ consists of disjoint simple cycles and simple paths, whose ends are the room-partitionings.

Of course Theorem 2.1 is Theorem 4.1 where all the manifolds of $M$ are the same, and the Theorem 3.5 is where $h=2$.

\section{Generalization to $d$-oiks}

A $d$-oik $C=(V, F), d \geq 1$, recently introduced by Edmonds [4], is a finite set $V$ of elements, called the vertices of $C$, and a family of subsets of $V$ each of size $d+1$, called the rooms of $C$, satisfying the following property: every subset of $V$ of $d$ elements is in an even number of the rooms. A wall of a room is a set obtained by deleting one vertex of the room, and so any wall of a room in an oik is the wall of a positive even number of rooms.

Let $M=\left[\left(V, F_{i}\right): i=1, \ldots, h\right]$ be an indexed collection of oiks, called an oik-family, all on the same vertex-set $V$. A room-family $R=\left[R_{i}: i=1, \ldots, h\right]$, 
for oik-family $M$, is where, for each $i, R_{i}$ is a room of oik $\left(V, F_{i}\right)$. A roompartitioning $R$ for $M$ is a room-family whose rooms partition $V$.

The concept of oik clearly generalizes the definition of simplicial pseudo manifold. The unifying Theorem 4.1 can then also be seen as an instance of the following theorem [4]:

Theorem 5.1 Given any oik-family $M$, there is an even number of roompartitionings for $M$.

Given a room-partitioning for $M$, an exchange algorithm can find another room-partitioning for $M$, once again by replacing one room by another. Our Corollary 2.3 shows that this algorithm is not a polynomial-time algorithm even for explicit (planar) 2-oiks, answering an open question in [4]. It would be interesting to characterize oiks for which an exchange algorithm is always well-bounded in the number of rooms.

\section{References}

[1] Cameron, K., and Edmonds, J., Existentially polytime theorems, DIMACS Series Discrete Mathematics and Theoretical Computer Science 1 (1990), 83-99.

[2] Cameron, K., and Edmonds, J., Some graphic use of an even number of odd nodes, Annales de l'Institut Fourier 49 (1999), 815-827.

[3] Chen, X., and Deng, X., Settling the complexity of two-player Nash equilibrium, Proceedings of the 47th Annual IEEE Symposium on Foundations of Computer Science (2006), 261-272.

[4] Edmonds, J., Euler complexes, in: Research Trends in Combinatorial Optimization, W. Cook, L. Lovasz and J. Vygen, editors, Springer, Berlin (2009), 65-68.

[5] Lemke, C.E., and Howson, J.T., Equilibrium points of bimatrix games, Journal of the Society for Industrial and Applied Mathemathics 12 (1964), 413-423.

[6] Morris, W., Lemke Paths on Simple Polytopes, Mathematics of Operations Research 19 (1994), 780-789.

[7] Papadimitriou, C.H., On the complexity of the parity argument and other inefficient proofs of existence, Journal of Computer and System Sciences $\mathbf{4 8}$ (1994), 498-532.

[8] Savani, R., and Von Stengel, B., Hard-to-solve bimatrix games, Econometrica 74 (2006), 397-429. 